\title{
Long-term maternal high-fat feeding from weaning through pregnancy and lactation predisposes offspring to hypertension, raised plasma lipids and fatty liver in mice
}

\author{
Maqsood M. Elahi ${ }^{1}$, Felino R. Cagampang ${ }^{1}$, Dhea Mukhtar ${ }^{1}$, Frederick W. Anthony ${ }^{1}$, Sunil K. Ohri ${ }^{2}$ \\ and Mark A. Hanson ${ }^{1} *$ \\ ${ }^{1}$ Institute of Developmental Sciences, Developmental Origins of Health and Disease Division, University of Southampton \\ School of Medicine, Southampton General Hospital, Mailpoint 887, Southampton SO16 6YD, UK \\ ${ }^{2}$ Wessex Cardiothoracic Centre, University of Southampton School of Medicine, Southampton General Hospital, Southampton \\ SO16 OYD, UK \\ (Received 13 June 2008 - Revised 22 October 2008 - Accepted 1 December 2008 - First published online 10 February 2009)
}

In rodents, adverse prenatal nutrition, such as a maternal diet rich in fat during pregnancy, enhances susceptibility of the offspring to hypertension, type 2 diabetes and other features of the human metabolic syndrome in adulthood. However, previous experimental studies were confined to shortterm modifications of the maternal diet during pregnancy and/or lactation periods, a situation uncommon in humans. Moreover in humans, the offspring may also consume a high-fat diet, which may take them beyond the range to which their development has adapted them to respond healthily. We examined in C57 mice the effects on offspring of feeding their mothers a high-fat (HF) or standard chow (C) diet from weaning through pregnancy and lactation, and whether there are additive phenotypic effects of feeding the offspring an HF diet from weaning to adulthood (dam-offspring dietary group HF-HF). This group was compared with offspring from HF-fed dams fed a C diet from weaning to adulthood (HF-C) and offspring from C-fed mothers fed the $\mathrm{C}$ or HF diet (C-C and HF-C, respectively). HF-HF, HF-C and C-HF adult female offspring were heavier, fatter, and had raised serum cholesterol and blood pressure compared with $\mathrm{C}-\mathrm{C}$ female offspring. We observed a similar trend in male offspring except for the HF-C group which was not heavier or fatter than male C-C offspring. Histology showed lipid vacuoles within hepatocytes in the HFHF, HF-C and C-HF but not the CC offspring. Serum C-reactive protein was elevated in female (C-HF and HF-HF) but not in male offspring. Elevated blood pressure in the HF-C and C-HF groups was attenuated in the HF-HF group in males but not in females. These findings indicate that long-term consumption of an HF diet by the mother predisposes her offspring to developing a metabolic syndrome-like phenotype in adult life, although cardiovascular effects of an HF diet are related to sex specificity in the HF-HF group.

Hypertension: Pregnancy: Diet: Obesity: Metabolic syndrome

In humans it has been well documented that transition from an environment where food is poor or adequate to one where diet is high in fat and carbohydrates is associated with higher prevalence of the metabolic syndrome, typified by type 2 diabetes, hypertension, hypercholesterolaemia and obesity ${ }^{(1-6)}$. It has been suggested that such a 'nutritional transition' to a Western diet may have deleterious consequences on the health of future generations by affecting early development, influencing susceptibility to disease in later life. We recently proposed that the extent of such susceptibility depends on the degree of mismatch between the developmental and later environments, for example, in nutritional content ${ }^{(7)}$.

Developmental induction of cardiovascular and metabolic risk factors has been observed in rodent models in which the pregnant dam is exposed to nutrient restriction and the offspring fed a normal chow diet ${ }^{(8-10)}$. However, there are fewer studies that have examined adaptive and maladaptive mechanisms during fetal development when pregnant dams are exposed to overnutrition. The issue is particularly important in the light of the increasing consumption of refined foods with a high glycaemic index and fat content among men and women now consuming $30 \%$ more saturated fats than the recommended daily intake ${ }^{(11)}$. Several studies, including ours, have shown in rats that a maternal diet rich in fat during pregnancy results in features of the metabolic syndrome such as obesity, sedentary behaviour and vascular dysfunction in the offspring ${ }^{(12-15)}$. However, these studies were confined to short-term modifications in the maternal diet during pregnancy and/or lactation periods alone. Altering the maternal diet during critical periods of gestation ${ }^{(16,17)}$ or throughout gestation and/or the suckling period ${ }^{(18,19)}$ results in a varying degree of phenotypic outcomes associated with the metabolic syndrome, suggesting the importance of the timing and duration of the nutritional insult. In humans, 
consumption of a high-fat diet just during pregnancy or suckling is not common, but there is increasing concern about the effects of obesogenic diets in children on the health of their future offspring. We therefore extended our animal model to investigate the consequences for their offspring of feeding dams a high-fat (HF) diet from the time that they were weaned, through their pregnancy and lactation until their pups are weaned. In addition we determined whether there are additive effects of feeding these offspring themselves an $\mathrm{HF}$ diet from weaning to adulthood.

\section{Methods \\ Experimental protocol}

All animal procedures were in accordance with the UK Animals (Scientific Procedures) Act 1986. Female C57BL/6 mice (Charles River Laboratories, Margate, Kent, UK) were maintained under a $12 \mathrm{~h}$ light-dark cycle at constant temperature $\left(25 \pm 2^{\circ} \mathrm{C}\right)$ with food and water available ad libitum. At age 4 weeks they were randomly allocated to either a control diet of standard laboratory chow $(\mathrm{C} ; 5.3 \%$ fat (maize oil), $21.2 \%$ protein and $49.2 \%$ carbohydrate; Special Diet Services, Witham, Essex, UK) or an HF experimental diet supplemented $18 \%(\mathrm{w} / \mathrm{w})$ with animal lard with additional vitamins and minerals, protein and choline to correct for the dilution (final composition in $\mathrm{g} \%$ (w/w): lard, 17.8; casein, 26.5; choline chloride, 0.3 ; L-cysteine, 0.4 ; rice starch, 28.3 ; cellulose, $6 \cdot 1$; soya oil, $4 \cdot 3$; sucrose, $10 \cdot 4$; minerals, $4 \cdot 3$; vitamins, 1.2; Special Diet Services diet no. 824053, Witham, Essex, UK). This HF diet is as used in previous studies ${ }^{(20)}$. At age 10 weeks, the females were time-mated and, after
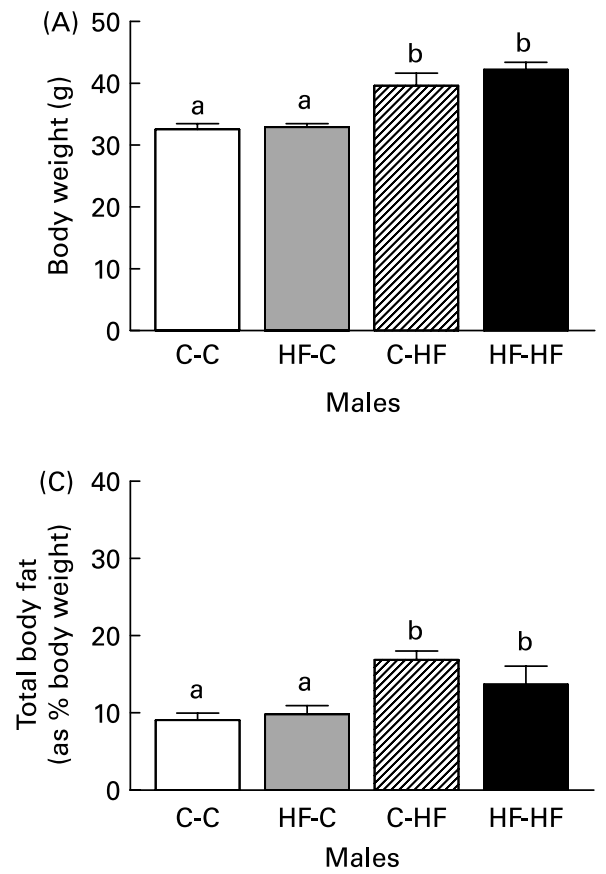

confirmation of mating (i.e. presence of vaginal plug), were individually housed under a $12 \mathrm{~h}$ light-dark cycle at constant temperature with water available ad libitum.

After birth, pups were weighed and litter size was reduced to eight pups and, when possible, to equal numbers of males and females. From weaning ( $21 \mathrm{~d}$ post-partum) offspring from the $\mathrm{HF}$ and $\mathrm{C}$ dams were fed either $\mathrm{HF}$ or $\mathrm{C}$ diets. We refer to the offspring born to HF dams as HF-HF and HF-C according to their post-weaning diet. Similarly, offspring born to $\mathrm{C}$ dams are referred to as $\mathrm{C}-\mathrm{HF}$ and $\mathrm{C}-\mathrm{C}$ according to their postweaning diet. Food intake and body weights were monitored weekly until the offspring reached adulthood. Adult offspring were killed at age 36 weeks by cervical dislocation. Blood was collected by cardiac puncture and fat depots (i.e. gonadal, retroperitoneal, interscapular, inguinal and peri-renal) were dissected and weighed. Cumulative fat depot weights for each animal were compared with total body weights and body fat as a percentage of total body weight was calculated. The livers were also dissected, fixed in $10 \%$ neutral buffered formaldehyde and stored for further histological analyses.

\section{Blood pressure measurements}

Systolic arterial blood pressure (BP) was measured by tail-cuff plethysmography, as described previously by Krege et al. ${ }^{(21)}$ who showed that BP taken by this method was highly correlated with intra-arterial BP measured by telemetry in unrestrained, unanesthetised animals ${ }^{(21)}$. We conducted the measurements in a heated room $\left(27-28^{\circ} \mathrm{C}\right)$ in order to get optimal BP readings at the same time during the day. All animals were made accustomed to the procedure for $7 \mathrm{~d}$ before
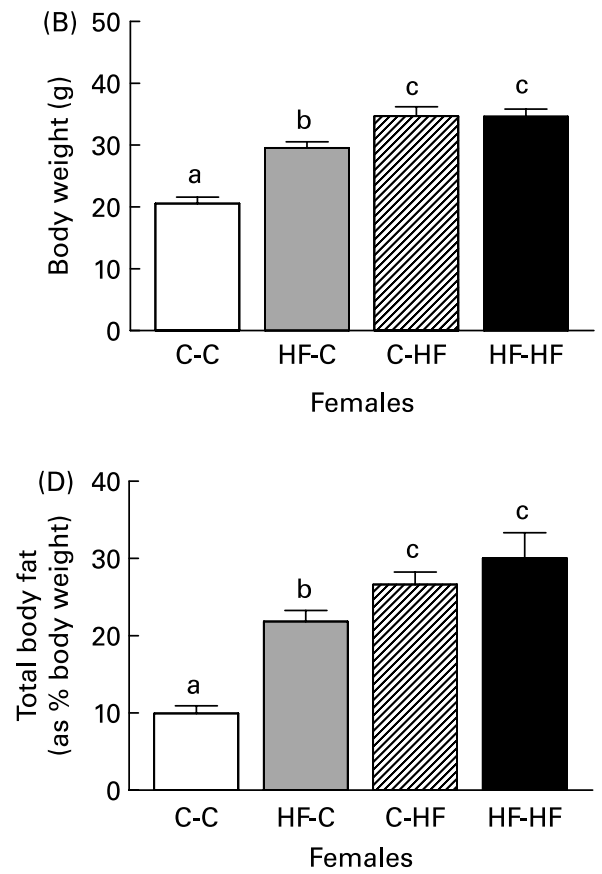

Fig. 1. Comparison of body weight $(A, B)$ and total body fat $(C, D)$ in male $(A, C)$ and female $(B, D)$ offspring from control-fed mothers that were then fed a chow diet $(\mathrm{C}-\mathrm{C})$ or a high-fat diet $(\mathrm{C}-\mathrm{HF})$ and from high-fat-fed mothers that were then fed a high-fat diet (HF-HF) or a chow diet (HF-C). Values are means $(n 8-10$ per group), with standard errors represented by vertical bars. ${ }^{a, b, c}$ Mean values with unlike letters were significantly different $(P<0 \cdot 05$; Tukey-Kramer comparisons test). 
each BP measurement session. At least five readings were taken from each animal per session and averaged to get a single session value. BP was measured at 13, 18, 23, 27, 30 and 36 weeks post-weaning. At each time point, we took the average BP values from eight offspring of each sex picked randomly from each of the eight litters in each treatment group.

\section{Measurement of plasma C-reactive protein and cholesterol}

$\mathrm{C}$-reactive protein (CRP) in the serum was measured using a sensitive double-antibody sandwich immunoassay enzymic ELISA rabbit anti-human CRP and peroxidase-conjugated rabbit anti-mouse CRP (VITROS CRP Slides; Vitros Products, Rochester, NY, USA) ${ }^{(22)}$. Monoclonal anti-CRP antibody conjugated to horseradish peroxidase served as the signal generator. The assay was linear up to $5 \mathrm{mg} / \mathrm{l}$ and logarithmic thereafter. The inter-assay CV were less than $10 \%$ across the range of measured results. Serum total cholesterol was measured with commercially available kits (Vitros Products) using enzymic methods and measured by reflectance spectrophotometry as previously reported ${ }^{(15,18)}$.

\section{Histology}

Fixed liver tissues were embedded in paraffin and 5-10 $\mu \mathrm{m}$ sections were cut and mounted on glass slides. Deparaffinised, fixed sections were stained with haematoxylin and eosin. Microscopic examination was performed on stained liver sections from representative animals in each group.

\section{Data analysis}

The biochemical and biophysical parameters in dams were analysed using one-way ANOVA followed by the TukeyKramer comparisons test. All data are expressed as mean values with their standard errors. A $P$ value less than 0.05 was considered to be statistically significant. All statistical analysis was calculated with SPSS 14.0 (SPSS, Inc., Chicago, IL, USA).
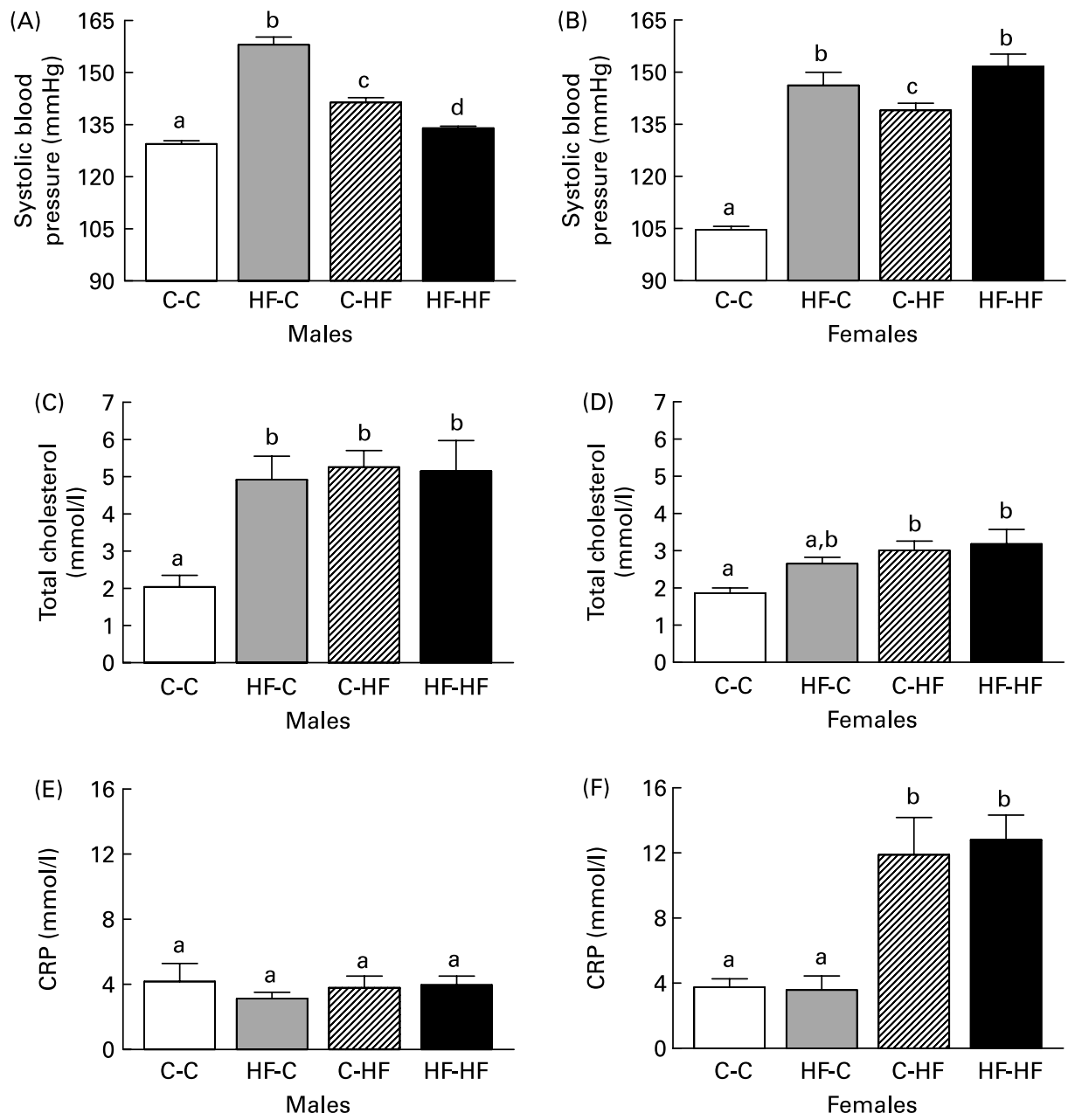

Fig. 2. Comparison of systolic blood pressure (A, B), total cholesterol $(C, D)$ and $C$-reactive protein (CRP) levels (E, F) in male $(A, C, E)$ and female $(B, D, F)$ offspring from control-fed mothers that were then fed a chow diet (C-C) or a high-fat diet (C-HF) and from high-fat-fed mothers that were then fed a high-fat diet (HF-HF) or a chow diet (HF-C). Values are means ( $n$ 8-10 per group), with standard errors represented by vertical bars. ${ }^{\text {a,b,c }}$ Mean values with unlike letters were significantly different $(P<0.05$; Tukey-Kramer comparisons test). 


\section{Results}

\section{Body weight and adiposity}

We did not find any significant difference in food intake among the various treatment groups during the experimental period (data not shown). HF-HF and C-HF male offspring were heavier at 36 weeks post-weaning than $\mathrm{HF}-\mathrm{C}$ and $\mathrm{C}-\mathrm{C}$ males (Fig. 1 (A)). These changes were also reflected in their total body fat (as percentage body weight), where the HF-HF and C-HF males had greater fat depots compared with the C-C and HF-C groups (Fig. 1 (C)). In female offspring the HF-C offspring were lighter than the HF-HF and $\mathrm{C}-\mathrm{HF}$ animals but heavier compared with the C-C animals (Fig. 1 (B)). Corresponding increases in total body fat were observed in these groups compared with the C-C females (Fig. 1 (D)).

\section{Blood pressure}

Systolic BP was elevated in the HF-HF, HF-C and C-HF male and female offspring at 36 weeks post-weaning compared with C-C offspring (Fig. 2 (A) and (B)). The HF-C males had the highest $\mathrm{BP}$ and this was significantly greater than the HF$\mathrm{HF}$ and C-HF groups. In the females, the HF-HF and HF-C groups had significantly elevated BP levels compared with the C-HF offspring.

\section{C-reactive protein and cholesterol levels}

Total cholesterol was elevated in the HF-HF, HF-C and C-HF males at 36 weeks post-weaning compared with the $\mathrm{C}-\mathrm{C}$ males (Fig. 2 (C)). Interestingly, no difference was observed in CRP levels among these four treatment groups (Fig. 2 (E)).
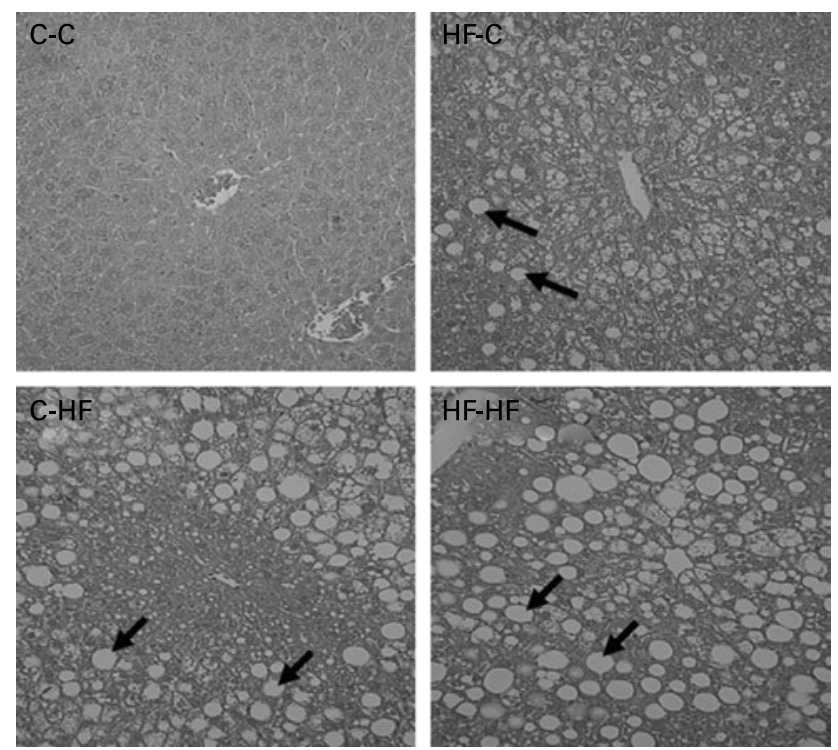

Fig. 3. Liver histology in female offspring from control-fed mothers that were then fed a chow diet (C-C) or a high-fat diet (C-HF) and from high-fatfed mothers that were then fed a high-fat diet (HF-HF) or a chow diet (HF-C). $\mathrm{C}-\mathrm{C}$ offspring had normal liver structure. However, lipid vacuoles $(\leftarrow)$ of various sizes could be observed within hepatocytes of the C-HF, HF-HF and HF-C offspring. Staining with haematoxylin and eosin; magnification $\times 20$; bar scale $=40 \mu \mathrm{m}$.
In females, total cholesterol and CRP level were elevated in the HF-HF and C-HF animals compared with the C-C offspring (Fig. 2 (D) and Fig. 2 (F), respectively). Total cholesterol in the HF-C group was also elevated but was not significantly different from the $\mathrm{C}-\mathrm{C}$ or from the $\mathrm{C}-\mathrm{HF}$ and HF-HF groups.

\section{Development of fatty liver in adult offspring}

Histological examination of the liver showed that the C-C offspring had normal liver structure (Fig. 3). However, we observed lipid vacuoles of various sizes within hepatocytes of the C-HF, HF-HF and HF-C offspring. Moreover, mononuclear cell infiltration, pyknotic nuclei and the rupturing of the endothelium of some central veins were observed in the livers in these groups of offspring compared with C-C offspring (data not shown).

\section{Discussion}

Nutritional status during critical periods of early life has important influences on development, and modification of the quality and/or quantity of maternal nutrition during pregnancy has been shown to have consequences on the later health of the offspring, changing their responses to environmental challenges and thus their predisposition to disease $^{(23,24)}$. In the present study we examined in a mouse model the consequences to the offspring of a long-term maternal HF dietary regimen, starting from when the prospective mothers were themselves weaned until weaning of their offspring. This contrasts with earlier animal experiments, which focused on the consequences for the offspring of maternal HF feeding during only gestation and/or lactation periods ${ }^{(12,13,15)}$. The long-term HF feeding results in changes in the dam's physiology, including increased body weight and raised circulating total and LDL-cholesterol levels compared with the C-fed group, as observed in our previous study ${ }^{(25)}$. These changes in the HF dams may provide 'cues' used by the developing offspring in altering phenotype in anticipation of their postnatal environment. The long-term dietary regimen used in the present study may better represent the situation in human populations, following socio-economic transitions where consumption of high-fat food occurs very early in life and continues in women through pregnancy and lactation. The present study demonstrates that offspring of such mothers are predisposed to becoming fat, hypercholesterolaemic and hypertensive in adulthood, thus perpetuating the cycle of chronic disease.

We previously reported that prenatal and early postnatal exposure to a maternal diet rich in animal fat leads to the development of characteristics similar to the human metabolic syndrome in adult rats, even when they are reared on a balanced diet ${ }^{(13,14)}$. The present study clearly shows that long-term consumption of an HF diet by the female dams predisposes their offspring to obesity, hypercholesterolaemia, hypertension and fatty liver in adult life, at least when they were also fed an HF diet. Greater adiposity was also seen in females, even when they were fed a $\mathrm{C}$ diet post-weaning, suggesting that predisposition to obesity had been induced during development. This was not, however, seen in the HF-C male offspring. 
In males the elevation of $\mathrm{BP}$ was less pronounced in the HF-HF group than in the HF-C or C-HF groups. This supports the partially beneficial cardiovascular effect of reducing the dietary mismatch in the HF-HF offspring reported in our earlier study ${ }^{(13)}$ although in that study the dams were only fed the HF diet during pregnancy and weaning, and endothelial dysfunction rather than elevated $\mathrm{BP}$ was attenuated compared with the C-fed offspring. These data are broadly in support of the predictive adaptive response concept ${ }^{(13)}$. However, as outlined in the original exposition of the concept ${ }^{(3)}$, such an effect only operates within a range of postnatal environments, beyond which the risk of pathophysiology is increased. The postnatal HF used in the present study is likely to take the offspring into such a range, one which from an evolutionary point of view is novel, possibly explaining the pathophysiological effects observed in them.

We observed a substantial number of lipid vacuoles within hepatocytes of the HF-HF, HF-C and C-HF but not the C-C offspring. Previous studies in rats have demonstrated that offspring of fat-fed dams have profound metabolic defects such as increased liver weight and liver TAG content ${ }^{(26,27)}$. It has been reported in humans that there is a strong association between hypercholesterolaemia, steatohepatitis (non-alcoholic fatty liver) and increased risk of $\mathrm{CVD}^{(28)}$ but it is unclear whether certain diets are more likely to produce these effects than others. Steatohepatitis and atherosclerosis are both produced in apo-E lipoprotein-deficient mice, suggesting a pathophysiological effects on both the liver and aorta of cholesterol-enriched diets ${ }^{(29,30)}$. The accumulation of lipids in hepatocytes suggests possible interference with mitochondrial and microsomal function leading to disruption in lipoprotein transport and fatty acid accumulation $v$. metabolism $^{(14)}$. The accumulation of lipids in hepatocytes in the HF-C offspring suggests that susceptibility to development of fatty liver can be induced during early development.

Chronic inflammation is a major contributor to atherosclerosis and $\mathrm{CVD}^{(31)}$. An important marker of inflammation is an elevation in CRP, an acute-phase reactant secreted by hepatocytes in response to pro-inflammatory cytokines such as IL-6 ${ }^{(32)}$. In several large epidemiological studies, CRP has been shown to be a strong, independent predictor of CVD risk in both men and women ${ }^{(33,34)}$. The mechanism by which inflammation increases CVD risk is not known, but during periods of acute inflammation lipid metabolism is altered, giving a a proatherogenic profile ${ }^{(35)}$. This is what we observed in our HF-HF and C-HF female offspring, which had elevated CRP levels. Interestingly, we did not find such increases in the male offspring, suggesting that the effect of HF exposure on CRP levels is sex specific. The increase in CRP levels found only in female offspring on the HF diet may be a consequence of increased sensitivity to the HF diet brought about by circulating female sex hormones $^{(34,36)}$ or due to the fact that the HF diet itself may result in high CRP in females. This observation is supported by human studies where CRP levels tend to be higher in women than in men ${ }^{(33,34,37-39)}$.

\section{Perspectives}

The current findings indicate that long-term consumption of an HF diet by the mother predisposes her offspring to obesity, hypercholesterolaemia, hypertension and development of fatty liver in adult life. This adverse effect on the offspring is induced during development and is not necessarily or completely reversed by either consumption of a postnatal $\mathrm{C}$ diet or indeed an HF diet. The results from the present study provide an experimental basis for investigating consequences of dietary transitions relevant to humans, where the woman's diet both before and during pregnancy and lactation may be a contributing factor to the development of metabolic and cardiovascular disease in her children.

\section{Acknowledgements}

We appreciate the technical assistance provided by Ronald Lee (Histochemistry Research Unit, University of Southampton) in performing the liver histology. Authors contributions are as follows: M. M. E. conducted the animal experiments and analysed the data; F. R. C. helped design the study and analysed the data; F. W. A. and D. M. assisted with laboratory analysis; S. K. O. helped design the study and M. A. H. designed the study and assisted with analysis. All authors were involved in writing the paper.

The present study was supported by the British Heart Foundation. M. M. E. is supported by a BUPA/HOPE research fellowship.

There are no conflicts of interest to declare.

\section{References}

1. Bateson P, Barker D, Clutton-Brock T, et al. (2004) Developmental plasticity and human health. Nature 430, 419-421.

2. Benyshek DC, Martin JF \& Johnston CS (2001) A reconsideration of the origins of the type 2 diabetes epidemic among Native Americans and the implications for intervention policy. Med Anthropol 20, 25-64.

3. Gluckman PD \& Hanson MA (2004) Living with the past: evolution, development, and patterns of disease. Science 305, $1733-1736$.

4. Kuzawa CW, Adair LS, Avila JL, et al. (2003) Atherogenic lipid profiles in Filipino adolescents with low body mass index and low dietary fat intake. Am J Hum Biol 15, 688-696.

5. Stookey JD, Adair L, Stevens J, et al. (2001) Patterns of longterm change in body composition are associated with diet, activity, income and urban residence among older adults in China. J Nutr 131, 2433S-2440S

6. Vickers MH, Breier BH, Cutfield WS, et al. (2000) Fetal origins of hyperphagia, obesity, and hypertension and postnatal amplification by hypercaloric nutrition. Am J Physiol Endocrinol Metab 279, E83-E87.

7. Gluckman P \& Hanson MA (2005) The Fetal Matrix: Evolution, Development and Disease. Cambridge, UK: Cambridge University Press.

8. Desai M, Byrne CD, Zhang J, et al. (1997) Programming of hepatic insulin-sensitive enzymes in offspring of rat dams fed a protein-restricted diet. Am J Physiol 272, G1083-G1090.

9. Franco MC, Arruda RM, Dantas AP, et al. (2002) Intrauterine undernutrition: expression and activity of the endothelial nitric oxide synthase in male and female adult offspring. Cardiovasc Res 56, 145-153.

10. Ozaki T, Nishina H, Hanson MA, et al. (2001) Dietary restriction in pregnant rats causes gender-related 
hypertension and vascular dysfunction in offspring. $J$ Physiol 530, $141-152$.

11. Food Standards Agency (2006) National Diet and Nutrition Survey: Young people aged 4 to 18 years: Revised consumption data for some food groups. http://www.food.gov.uk/science/ dietarysurveys/ndnsdocuments/ndnssurvey4to18

12. Bayol SA, Simbi BH \& Stickland NC (2005) A maternal cafeteria diet during gestation and lactation promotes adiposity and impairs skeletal muscle development and metabolism in rat offspring at weaning. J Physiol 567, 951-961.

13. Khan I, Dekou V, Hanson M, et al. (2004) Predictive adaptive responses to maternal high-fat diet prevent endothelial dysfunction but not hypertension in adult rat offspring. Circulation 110, 1097-1102.

14. Khan IY, Taylor PD, Dekou V, et al. (2003) Gender-linked hypertension in offspring of lard-fed pregnant rats. Hypertension 41, 168-175.

15. Levin BE \& Govek E (1998) Gestational obesity accentuates obesity in obesity-prone progeny. Am J Physiol 275, R1374-R1379.

16. Langley-Evans SC, Clamp AG, Grimble RF, et al. (1996) Influence of dietary fats upon systolic blood pressure in the rat. Int $J$ Food Sci Nutr 47, 417-425.

17. Zambrano E, Bautista CJ, Deas M, et al. (2006) A low maternal protein diet during pregnancy and lactation has sex- and window of exposure-specific effects on offspring growth and food intake, glucose metabolism and serum leptin in the rat. $J$ Physiol 571, 221-230.

18. Khan IY, Dekou V, Douglas G, et al. (2005) A high-fat diet during rat pregnancy or suckling induces cardiovascular dysfunction in adult offspring. Am J Physiol Regul Integr Comp Physiol 288, R127-R133.

19. Zhang J, Wang C, Terroni PL, et al. (2005) High-unsaturatedfat, high-protein, and low-carbohydrate diet during pregnancy and lactation modulates hepatic lipid metabolism in female adult offspring. Am J Physiol Regul Integr Comp Physiol 288, R112-R118.

20. Van Heek M, Compton DS, France CF, et al. (1997) Dietinduced obese mice develop peripheral, but not central, resistance to leptin. J Clin Invest 99, 385-390.

21. Krege JH, Hodgin JB, Hagaman JR, et al. (1995) A noninvasive computerized tail-cuff system for measuring blood pressure in mice. Hypertension 25, 1111-1115.

22. Danesh J, Wheeler JG, Hirschfield GM, et al. (2004) C-reactive protein and other circulating markers of inflammation in the prediction of coronary heart disease. $N$ Engl J Med 350, $1387-1397$

23. Langley-Evans SC (1996) Intrauterine programming of hypertension in the rat: nutrient interactions. Comp Biochem Physiol A Physiol 114, 327-333.
24. Oh W, Gelardi NL \& Cha CJ (1988) Maternal hyperglycemia in pregnant rats: its effect on growth and carbohydrate metabolism in the offspring. Metabolism 37, 1146-1151.

25. Elahi MM, Cagampang FR, Anthony FW, et al. (2008) Statin treatment in hypercholesterolemic pregnant mice reduces cardiovascular risk factors in their offspring. Hypertension 51, 939-944.

26. Guo F \& Jen KL (1995) High-fat feeding during pregnancy and lactation affects offspring metabolism in rats. Physiol Behav 57, 681-686.

27. Koukkou E, Ghosh P, Lowy C, et al. (1998) Offspring of normal and diabetic rats fed saturated fat in pregnancy demonstrate vascular dysfunction. Circulation 98, 2899-2904.

28. Reid AE (2001) Nonalcoholic steatohepatitis. Gastroenterology 121, 710-723.

29. Shiri-Sverdlov R, Wouters K, van Gorp PJ, et al. (2006) Early diet-induced non-alcoholic steatohepatitis in APOE2 knock-in mice and its prevention by fibrates. $J$ Hepatol 44, 732-741.

30. Tous M, Ferre N, Camps J, et al. (2005) Feeding apolipoprotein E-knockout mice with cholesterol and fat enriched diets may be a model of non-alcoholic steatohepatitis. Mol Cell Biochem 268, $53-58$.

31. Libby P (2002) Inflammation in atherosclerosis. Nature 420, $868-874$.

32. Libby P, Willerson JT \& Braunwald E (2004) C-reactive protein and coronary heart disease. $N$ Engl J Med 351, 295-298.

33. Ridker PM, Cushman M, Stampfer MJ, et al. (1997) Inflammation, aspirin, and the risk of cardiovascular disease in apparently healthy men. $N$ Engl J Med 336, 973-979.

34. Ridker PM, Hennekens CH, Buring JE, et al. (2000) C-reactive protein and other markers of inflammation in the prediction of cardiovascular disease in women. $N$ Engl J Med 342, 836-843.

35. Khovidhunkit W, Kim MS, Memon RA, et al. (2004) Effects of infection and inflammation on lipid and lipoprotein metabolism: mechanisms and consequences to the host. J Lipid Res $\mathbf{4 5}$, 1169-1196.

36. Ridker PM, Hennekens CH, Rifai N, et al. (1999) Hormone replacement therapy and increased plasma concentration of C-reactive protein. Circulation 100, 713-716.

37. Visser M, Bouter LM, McQuillan GM, et al. (1999) Elevated C-reactive protein levels in overweight and obese adults. JAMA 282, 2131-2135.

38. Ridker PM, Cushman M, Stampfer MJ, et al. (1997) Inflammation, aspirin, and the risk of cardiovascular disease in apparently healthy men. $N$ Engl J Med 336, 973-979.

39. Ridker PM, Hennekens CH, Buring JE, et al. (2000) C-reactive protein and other markers of inflammation in the prediction of cardiovascular disease in women. $N$ Engl J Med 342, $836-843$ 